\title{
Dynamic Economic Dispatch for Microgrid Based on the Chance-Constrained Programming
}

\author{
Daizheng Huang*, Lingling $\mathrm{Xie}^{* *}$ and $\mathrm{Zhihui} \mathrm{Wu}^{\dagger}$
}

\begin{abstract}
The power of controlled generators in microgrids randomly fluctuate because of the stochastic volatility of the outputs of photovoltaic systems and wind turbines as well as the load demands. To address and dispatch these stochastic factors for daily operations, a dynamic economic dispatch model with the goal of minimizing the generation cost is established via chance-constrained programming. A Monte Carlo simulation combined with particle swarm optimization algorithm is employed to optimize the model. The simulation results show that both the objective function and constraint condition have been tightened and that the operation costs have increased. A higher stability of the system corresponds to the higher operation costs of controlled generators. These operation costs also increase along with the confidence levels for the objective function and constraints.
\end{abstract}

Keywords: Microgrid, Dynamic economic dispatch, Chance-constrained programming

\section{Introduction}

The large energy demand has driven the development of microgrids (MG). With their many potential system benefits such as reducing power losses from the grid, deferring grid capacity investments, reducing emissions, and reducing electricity generation costs, MGs have attracted wide attention for their efficient, flexible, intelligent control and their great potential in addressing issues in the environment, energy depletion, and power quality [1-4].

However, renewable power generations, such as photovoltaic (PV) systems and wind turbines (WT), that face uncertainties under natural conditions cannot supply a stable power output to satisfy the requirements of the load demand (LD). A power supply-demand balance must be achieved by using stable distributed power sources, including microturbines (MT), diesel generators (DG), fuel cells (FC), and electric power storages such as storage batteries (BT), in an isolated MG. Using fossil fuels in stable distributed power sources will emit harmful gases that reduce the economic and environmental benefits of wind power and solar energy. This case presents a critical issue for an isolated MG with a small capacity. Accordingly, the proper economic dispatch of MG becomes increasingly significant to achieve the following goals:

- Minimize the operation and maintenance costs;

- Reduce environmental effects; and

- Ensure the stable operation of the system and satisfy

$\dagger$ Corresponding Author: Dept of Biomedical Engineering, Guangxi Medical University, China. (923363211@qq.com)

* Dept of Biomedical Engineering, Guangxi Medical University, China. (daizheng-huang@qq.com)

** School of Electrical Engineering, Guangxi University, China. (xielingling1318@163.com)

Received: February 3, 2016; Accepted: March 13, 2017 the load demands.

Several studies have attempted modeling with uncertainties under changing demands and fluctuations in renewable energy generation [5-8]. Economic dispatch has been divided into static and dynamic economic dispatch. The objective function of static economic dispatch is obtained on a time section. This dispatch also considers the static constraints, but ignores the internal relations among different time sections [9, 11]. By contrast, the dynamic economic dispatch considers the internal relation among the time sections, thereby making this dispatch more suitable than static economic dispatch for the actual operations of the MG system [12-16].

Uncertain programming is a type of mathematical programming that involves uncertain variables. Chanceconstrained programming (CCP) is an important branch of uncertain programming that solves the stochastic optimization problem with confidence level and uncertainty [17]. The power of PV, WT, and LD must be treated as random instead of constant because of their fluctuating levels. A dynamic economic dispatch model was proposed based on CCP to address the uncertain factors of MG with renewable power generation. The probability constraint concerning MG reliability was appended in this model. A confidence level was also assigned to the spinning reserve constraint to satisfy the system requirements, while the minimum cost of controlled generators $(C G)$ no less than a specified confidence level was selected as the objective function. The possible inter-dependencies among random parameters, including LD and the outputs of WT and PV, were ignored. The particle swarm optimization (PSO) algorithm based on Monte Carlo (MC) simulation was applied to optimize the model.

The system structure of MG proposed by clean energy resource teams included $\mathrm{PV}, \mathrm{WT}, \mathrm{DG}, \mathrm{MT}, \mathrm{FC}$, and $\mathrm{BT}$ 
and was applied in the experiments. The operation was simulated for 24 hours $[18,19]$.

\section{Model}

\subsection{CCP}

$\mathrm{CCP}$ is mainly used to address those problems with random variables in constraint. This type of programming must make a decision before measuring these variables. Given that constraints may be broken in some untoward conditions, CCP allows the decision to violate constraints as long as the probability of the constraint conditions are satisfied and is no less than a specified confidence level $[17,20]$.

A typical mathematic programming model with stochastic parameters can be expressed as follows:

$$
\left\{\begin{aligned}
& \min \bar{f} \\
\text { s.t } \quad & P_{r}\{f(x, \zeta) \leq \bar{f}\} \geq \alpha \\
& \left\{g_{j}(x, \zeta) \leq 0, j=1,2, \ldots, p\right\} \geq \beta
\end{aligned}\right.
$$

where $x$ is the decision variable, $\zeta$ is the stochastic variable, $\operatorname{Pr}\{\cdot\}$ is the probability of $\{\cdot\}, f(x, \zeta)$ is the objective function, and $g_{j}(x, \zeta)$ is the function of stochastic constraint. $\alpha$ and $\beta$ are confidence levels that are decided by a decision maker and are applied to the objective function and the function of constraints, while $\bar{f}$ denotes the minimum objective function on the basis of the specified confidence level $\alpha$.

\subsection{Dynamic economic dispatch model of MG}

The economic dispatch model considers the active power and disregards the reactive power and voltage. In this study, a day is divided into 24 periods with one-hour intervals. The controlled microsources, including DG, $\mathrm{MT}$, and FC, are assumed to track rapidly the variations of LD, WT, and PV. The system can run smoothly in the islandmode. The random outputs of PV and WT cannot be controlled under natural conditions and do not require the consumption of fossil fuel. Therefore, the minimum cost of $\mathrm{CG}$ will be used as the objective function that is no less than a specified confidence level.

\subsection{Objective function}

The objective function is defined as follows:

$$
\left\{\begin{array}{c}
P\left\{\sum_{t=1}^{24} \sum_{j}^{3} C_{O M j}\left(P_{t j}\right)+C_{f t j}\left(P_{t j}\right)\right. \\
\left.+C_{e n t j}\left(P_{t j}\right)+C_{\mathrm{var} t j}\left(P_{t j}\right) \leq \bar{F}\right\} \geq \beta_{1}
\end{array}\right.
$$

Table 1. Emission coefficient of mcrosource(g/kW.h)

\begin{tabular}{c|c|c|c|c}
\hline MSs & $\mathrm{NO}_{\mathrm{x}}$ & $\mathrm{CO}_{2}$ & $\mathrm{CO}$ & $\mathrm{SO}_{2}$ \\
\hline DG & 4.3314 & 232.0373 & 2.3204 & 0.464100 \\
\hline $\mathrm{MT}$ & 0.6188 & 184.0829 & 0.1702 & 0.000928 \\
\hline $\mathrm{FC}$ & 0 & 635.04 & 0.0544 & 0 \\
\hline
\end{tabular}

Table 2. Environmental evaluation standard of pollutant in power industry

\begin{tabular}{c|c|c|c|c}
\hline Coefficient & $\mathrm{NO}_{\mathrm{x}}$ & $\mathrm{CO}_{2}$ & $\mathrm{CO}$ & $\mathrm{SO}_{2}$ \\
\hline Environmental value & 1.000 & 0.002875 & 0.125 & 0.750 \\
\hline Fine & 0.250 & 0.00125 & 0.020 & 0.125 \\
\hline
\end{tabular}

where $t$ is the scheduling time (a whole day), $j$ is a type of CG (i.e., DG, MT, and FC), and $P_{t j}$ is the active power of the $j$ MS on the $i$ th time and is taken as a decision variable for the model. $C_{O M t j}$ denotes the operation maintenance costs of the $j \mathrm{MS}$ on the $t$ th time where $C_{O M t j}=K_{O M j} P_{t j}$, while $K_{O M j}$ is the operation management coefficient of the $j$ MS under the unit output where $K_{O M}(D G)=$ $0.01258 \$ / \mathrm{kWh}, K_{O M}(M T)=0.00587 \$ / \mathrm{kWh}$, and $K_{O M}(F C)$ $=0.00419 \$ / \mathrm{kWh}[21] . C_{f t j}$ is the fuel cost of the $j \mathrm{MS}$ on the $t$ th time. In $C_{f t}(D G)=a P^{2}+b P+c, a, b$, and $c$ are constraint coefficients provided by the manufacturer where $a=0.0074, b=0.2333$, and $c=0.4333$ [22]. In $C_{f i}(M T, F C)=\left(C_{n g} P \Delta t\right) /\left(\eta Q_{L H V}\right), \quad P$ is the output of MT and FC, $C_{n g}$ is the unit price of natural gas where $C_{n g}=0.4 \$ / \mathrm{m}^{3}, \eta$ is the generating efficiency where $\eta(M T)=30 \%$ and $\eta(F C=55 \%), \Delta t$ is the scheduling time where $\Delta t=1 h, Q_{L H V}$ is the value of low energy of natural gas where $Q_{L H V}=9.7 \mathrm{~kW} \cdot \mathrm{h} / \mathrm{m}^{3}$, and $C_{e n t j}$ is the environmental conversion cost of the $j$ MS on the $t$ th time. In $C_{\text {envtj }}=\sum_{k=1}^{M} K_{\text {envk }} \cdot P_{i j} \cdot\left(V_{k}+V_{k}^{\prime}\right), K_{\text {envk }}$ is the discharge coefficient of the $K$ pollutant, $M$ is the type of pollutant, $V_{k}$ is the environmental value of the pollutant, and $V_{k}{ }^{\prime}$ is the fine for pollution. Tables 1 and 2 list the pollution emissions of $\mathrm{CG}$ and the annotation of evaluation, respectively [23]. $C_{\mathrm{var} t j}\left(\delta_{t j}\right)=\lambda_{j} \delta_{t j}$ is the power adjustment cost of the $j$ MS on the $t$ th time, $\delta_{t j}$ is the power fluctuation of the $j$ MS on the $t$ th time, $\lambda_{j}$ is the unit power adjustment cost of the $j \mathrm{MS}$, and $\beta_{1}$ is a specified confidence level, which physical significance is reflected in the minimum probability of a successful power adjustment. $\bar{F}$ denotes the minimum cost of CG when the probability of $P\{\cdot\}$ is no less than the specified confidence level $\beta_{1}$.

\subsection{Constraints}

\subsubsection{Capacity constraint}

Each output of MS is restricted by the lower and upper limits as follows:

$$
P_{i}^{\min }<P_{i}<P_{i}^{\max }, i=1, \ldots \ldots, N
$$


where $P_{i}^{\min }$ and $P_{i}^{\max }$ are the minimum and maximum powers of the i type of MS, respectively.

\subsubsection{Power balance constraint}

The power of the system must meet the following constraint to operate safely and stably:

$$
P_{L D t}-\left(P_{D G t}+P_{M T t}+P_{F C t}+P_{P V t}+P_{W T t}+P_{B T t}\right)=0
$$

where $P_{L D t}$ is the power of LD on the $t$ th time, $P_{D G t}$, $P_{M T t}, P_{F C t}, P_{W T t}$, and $P_{P V t}$ are the outputs of DG, MT, FC, WT, and PV on the $t$ th time, respectively, and $P_{B T t}$ is the charge or discharge power of BT on the $t$ th time.

\subsubsection{Ramp constraint of $C G$}

$$
R_{i}^{d} \leq P_{i t}-P_{i(t-1)} \leq R_{i}^{u}
$$

where $R_{i}^{d}$ and $R_{i}^{u}$ are the descending and ascending rates of power produced by $\mathrm{CG}$, respectively, while $P_{i t}$ and $P_{i(t-1)}$ are the outputs of CG on $t$ th and( $t$-1)th times, respectively.

\subsubsection{Charge and discharge constraint of $B T$}

The BT and other MSs will be supplied when the power of LD is greater than the sum output of WT and PV. When the sum output of WT and PV exceeds the load demand, the BT will charge.

When the BT is charging or discharging, two constraints must be satisfied. First, the maximum and minimum states of charge $\left(\mathrm{SOG}_{\max }\right.$ and $\mathrm{SOC}_{\min }$, respectively) must account for $100 \%$ and $20 \%$ of the BT capacity, respectively.

$$
S O C_{\min }<S O C<S O C_{\max }
$$

Second, the power of charging $\left(P_{+}\right)$and discharging $\left(P_{-}\right)$in the unit time must account for $20 \%$ of the rated power of the battery $\left(P_{B A T}\right)$ to extend its service life.

$$
\left\{\begin{array}{l}
P_{+} \leq 0.2 P_{B A T} \\
P_{-} \leq 0.2 P_{B A T}
\end{array} .\right.
$$

\subsubsection{Spinning reserve constraint}

$$
P\left\{P_{D G t}+P_{M T t}+P_{F C t}+P_{P V t}+P_{W T t}+P_{B T \max t}+P_{S R t} \geq P_{L D t}\right\} \geq \beta_{2}
$$

where $P_{B T t \max }$ is the maximum output of BT on the $t$ th time, $P_{S R t}$ is the spinning reserve of $\mathrm{MG}$ on the $t$ th time. In $P_{S R t}=\sum_{i=1}^{3} \min \left\{R_{i}^{u} \Delta \tau, P_{i \max }-P_{i t}\right\}, i$ is the type of $\mathrm{CG}$, $\Delta \tau$ takes 1 to 5 minutes in general, and $\beta_{2}$ is a specified confidence level. The physical significance of $\left(1-\beta_{2}\right)$ is reflected in the maximum probability of allowing loss of load and reflects the stability of the system.

\section{Randomness of PV, WT, and LD}

\subsection{Probability model of WT}

Statistical results show that the Weibull distribution can describe the random variation of wind speed [24, 25]. The distribution function $\mathrm{F}(v)$ of wind speed $(v)$ can be described as follows:

$$
F(v)=1-\exp \left[-(v / c)^{k}\right]
$$

where $k=\left(\frac{\sigma}{\mu}\right)^{-1.086}$ is the shape parameter, $c=\frac{\mu}{\Gamma\left(1+\frac{1}{k}\right)}$ is the scale parameter, $\mu$ is the average wind speed, $\sigma$ is the standard deviation of wind speed, and $\Gamma(\cdot)$ is the gamma function.

The probability density function of wind speed can be expressed as follows:

$$
f(v)=(k / c)(v / c)^{k-1} \exp \left[-(v / c)^{k}\right]
$$

The relationship between the output of WT and wind speed can be approximately expressed as follows:

$$
P_{W T}=\left\{\begin{array}{c}
0, \quad v<v_{c i} \text { or } v \geq v_{c o} \\
P_{r} \frac{v-v_{c i}}{v_{r}-v_{c i}}, v_{c i} \leq v<v_{r} \\
P_{r}, \quad v_{r} \leq v<v_{\text {out }}
\end{array}\right.
$$

where $P_{r}$ is the rated power of WT, while $v_{r}, v_{c i}, v_{c o}$ are the rated, cut in, and cut out of wind speed, respectively. These values are provided by the manufacturer as $P_{r}=10$ $\mathrm{kW}, v_{c i}=2.5 \mathrm{~m} / \mathrm{s}, v_{r}=12 \mathrm{~m} / \mathrm{s}$, and $v_{c o}=18 \mathrm{~m} / \mathrm{s}$.

The distribution function of $P_{W T}$ in the range of $(-\infty,+\infty)$ can be expressed as follows:

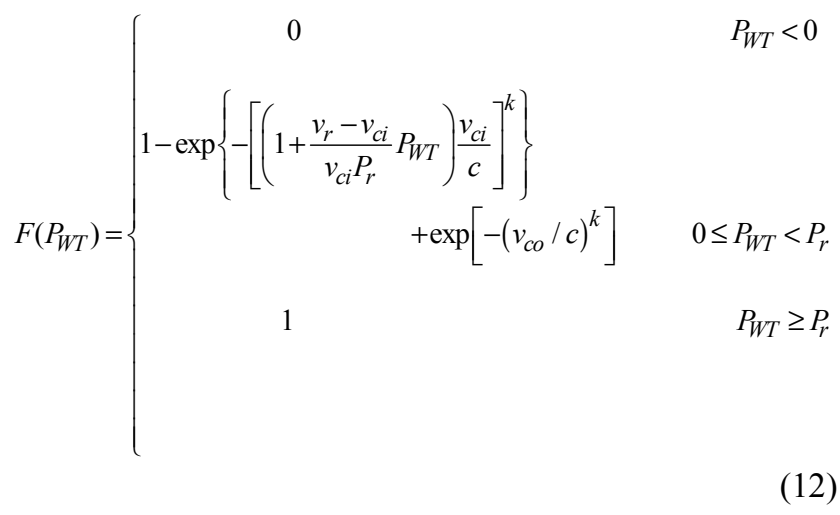

The power of WT is affected not only by wind speed, 
but also by altitude, air temperature and humidity, and blade pollution. The output power of WT will decrease accordingly with rising of the air temperature, vapour and altitude for reason that the air density and pressure atmosphere will decrease. The surface of the blade will change the roughness when it is polluted by dust, insects and oil leakage, which will make the aerodynamic characteristics of the airfoil change and reduce the power output [26].

The output of WT can be calculated as follows. First, the monthly average wind speed at the designated location can be obtained online using the HOMER software, and the hourly wind speed will be calculated [27, 28]. Second, the average and standard deviation of wind speed can be calculated according the hourly wind speed, and then $k$ and $c$ will be calculated. Third, the wind speed data will be produced via MC simulation, and the output of WT will be calculated according to formula (11).

\subsection{Probability model of PV}

The statistical results show that the beta distribution can approximately describe the solar light intensity within a relatively short period $[29,30]$. The probability density can be expressed as follows:

$$
f(E)=\frac{\Gamma(\alpha+\beta)}{\Gamma(\alpha) \Gamma(\beta)}\left(\frac{E}{E_{\max }}\right)^{\alpha-1}\left(1-\frac{E}{E_{\max }}\right)^{\beta-1}
$$

Where $E$ and $E_{\max }$ are the actual and maximum solar light intensities, respectively, $\alpha$ and $\beta$ are the shape parameters of beta distribution where $\alpha=\mu \cdot\left[\frac{\mu \cdot(1-\mu)}{\delta^{2}}-1\right]$ and $\beta=(1-\mu) \cdot\left[\frac{\mu \cdot(1-\mu)}{\delta^{2}}-1\right]$ in which $\mu$ and $\delta$ are the average and standard deviation of solar light intensity within a specified period, and $\Gamma$ is a gamma function.

The relationship between the output of PV and solar light intensity can be expressed as follows:

$$
P_{P V}=E A \eta
$$

where $A$ is the area of $\mathrm{PV}$ and $\eta$ is the photoelectric conversion efficiency.

In this case, the output of PV can be described as a beta function, which probability density can be expressed as follows:

$$
f\left(P_{P V}\right)=\frac{\Gamma(\alpha+\beta)}{\Gamma(\alpha) \Gamma(\beta)}\left(\frac{P_{P V}}{P_{\max }}\right)^{\alpha-1}\left(1-\frac{P_{P V}}{P_{\max }}\right)^{\beta-1}
$$

The maximum output can be expressed as $P_{\max }=$ $E_{\text {max }} A \eta$, where $P_{\text {max }}=80 \mathrm{~W}$.

The amount of solar radiation reaching the ground is mainly affected by solar altitude angle, latitude, atmospheric transparency, sunshine hours and altitude. The higher the solar elevation angle is, the greater the solar radiation intensity is. The solar elevation angle will change with latitude. The higher the latitude is, the smaller solar elevation angle is. The air gets thinner when the altitude is higher, and the solar radiation absorption and scattering is less. As a result, the higher the altitude is, the greater the solar radiation energy is [31].

The output of PV can be solved as follows. First, the hourly solar light intensity at the latitude and longitude of the designated location can be measured using HOMER [28]. Second, the average and standard deviation of solar light intensity can be calculated according to the hourly solar light intensity, and then $\alpha$ and $\beta$ will be calculated. Third, the solar light intensity density will be produced via MC simulation, and the output of PV will be calculated.

\subsection{Probability model of LD}

Practice has proven that the normal distribution can approximately reflect the stochastic of LD [32], which probability density function can be expressed as follows:

$$
f\left(P_{L D}\right)=\frac{1}{\sqrt{2 \pi} \sigma} \exp \left(-\frac{P_{L D}-\mu}{2 \sigma^{2}}\right)^{2}
$$

where $\mu$ and $\sigma$ are the average and standard deviation of LD, respectively, both of which can be calculated based on the historical LD data. Afterward, LD can be randomly generated according to formula (16).

The load is affected by the change of climate and temperature in different seasons and different regions.

\section{Optimization algorithm}

It can not to optimal random variable by PSO directly for the algorithm need fixed values of the parameters. In fact, the random variables and chance constraints introduced in the previous article cannot be easily translated into a deterministic optimization model. The proposed PSO algorithm combined with MC simulation can be used to solve this problem.

The MC simulation for optimization is applied to sample data from a known probability distribution of random variables. These data can then be used as the bases for making or testing decisions [33]. In this case, $\mathrm{MC}$ simulation is mainly used in formulas (2) and (8).

1) For the formula of objective function (2): Calculate the value of the objective function.

The random wind and solar output will lead to the stochastic output of CG. The independent random vector $\xi_{i}(i=1,2, \ldots, N)$ will be generated according to the probability distribution of wind, solar, and load as mentioned in Section 2. Set $F_{i}=F\left(P_{t j}, \xi_{i}\right)$ and sort $\left\{F_{1}, F_{2} \cdots, F_{N}\right\}$, then let $N^{\prime}=\left[N \beta_{1}\right]$. The $N^{\prime}$ th smallest 
number in $\left\{F_{1}, F_{2} \cdots, F_{N}\right\}$ will be used as the objective function $\bar{F}$ according to the law of large numbers.

2) For the spinning reserve constraint (8): Inspect the chance constraint.

Set $N^{\prime}=0$. The output $\left(P_{j t}\right)$ of MSs and LD will be generated according to their probability distributions. Take $P_{j t}$ into constraint (8) and determine whether $P_{j t}$ can meet the constraint. Let $N^{\prime} \leftarrow N^{\prime}+1$ when $P_{j t}$ meets the inequality (8), and then repeat this process $N$ times until $N^{\prime} / N \geq \beta_{2}$.

To inspect feasibility on the particles for the PSO, the equality constraint must be transferred into the inequality constraint. Each CG in equality (4) is placed on the left side of the equation, while the remaining MSs are placed on the right side as follows:

$$
P_{D G t}=\left(P_{M T t}+P_{F C t}+P_{P V t}+P_{W T t}+P_{B T t}\right)-P_{L D t}
$$

Afterward, $P_{D G t}$ in inequality (3) will be substituted by $P_{D G t}$ in equality (18) as follows:

$P_{D G t}^{\min }<\left(P_{M T t}+P_{F C t}+P_{P V t}+P_{W T t}+P_{B T t}\right)-P_{L D t}<P_{D G t}^{\max }$

The optimization process can be described as follows:

Step 1: Input the parameters of MSs, including the running, cost, and distribution of random variables. Set the confidence levels for the objective function and constraint;

Step 2: Input the parameters of the PSO algorithm, including particle size, maximum number of iterations, learning factor, particle velocity range, initial inertia weight, and final inertia weight;

Step 3: The power of WT, PV, and LD in each time is generated randomly based on the MC simulation according to the distribution presented in Section 3.

Step 4: The power of MT, DG, FC, and BT in each time is randomly generated within the range of the reactive power, that is, the power must satisfy inequality (3). MT, DG, FC, and BT are composed of population vectors;

Step 5: Test the feasibility of the population vectors. These vectors will be regenerated until they meet formulas (5) and (18). The population of BT must meet formulas (6) and (7) at the same time. The regeneration will be continued until the initial population vectors are completed. If the constraint has random variables such as the spinning reserve constraint (8), then the $\mathrm{MC}$ simulation technique described in step 2 will be applied to settle such variables. The initial velocity of the particles is randomly generated at the same time.

Step 6: Calculate the fitness of each particle via the stochastic simulation described in step 1 and then compare its fitness with the individual optimal value. If the fitness is better than the individual optimal value, then the current individual optimal value and position will be updated. The fitness will also be compared with the global optimal value. If the fitness is better than the global optimal value, then the current global optimal value and position will be updated;

Step 7: Update the velocity and position of each particle and test its feasibility according to formulas (5)-(8) and (18). If not feasible, then the velocity will be repeated to regenerate and its position will be updated; and

Step 8: Determine whether the operation iteration has reached its maximum number or not. If the operation iteration has reached its maximum, then the ultimate objective functions will be obtained. Calculate the power of each generating unit and the CG cost, and then exit the loop. Otherwise, go to Step 3 to continue.

\section{Simulation results}

\subsection{Specified data}

A model is simulated and analyzed to prove the validity of the method. This model is an island MG that is isolated from the utility grid, which includes PV, WT, DG, MT, FC, and BT. A whole day was taken as an example and separated into 24 periods with one-hour intervals. The outputs of WT, PV, and LD on a specified location during these 24 periods are generated via MC simulation as described in Sections 3.1 to 3.3. For simulation convenience and to consider the effect of stochastic

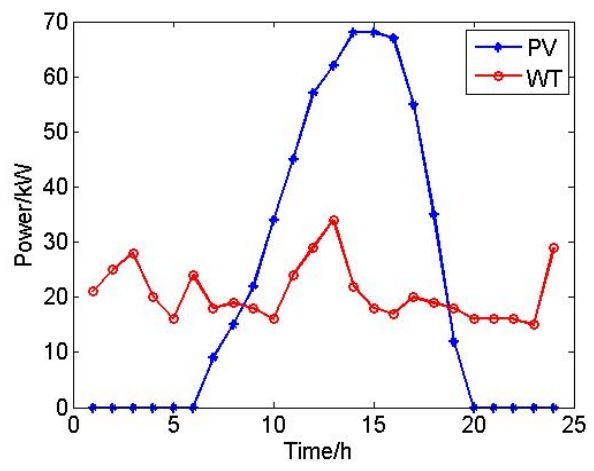

(a) Power of MT and PV

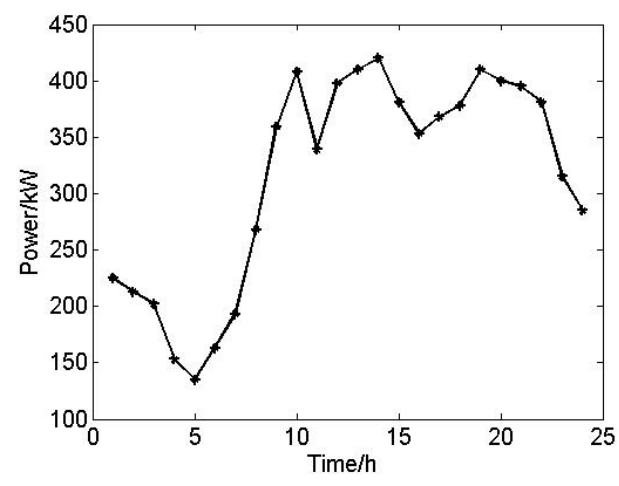

(b) Power of LD

Fig. 1. Power of MT, PV and LD 
Table 3. The parameters of the CG

\begin{tabular}{c|c|c|c|c}
\hline MSs & $\begin{array}{c}\text { Maximum } \\
\text { power(kW) }\end{array}$ & $\begin{array}{c}\text { Minimum } \\
\text { power(kW) }\end{array}$ & $\begin{array}{c}\text { Descending rate } \\
(\mathrm{kW} / \mathrm{min})\end{array}$ & $\begin{array}{c}\text { Rising rate } \\
(\mathrm{kW} / \mathrm{min})\end{array}$ \\
\hline MT & 60 & 5 & 1 & 1 \\
\hline FC & 90 & 10 & 2 & 2 \\
\hline DG & 300 & 20 & 1 & 1 \\
\hline
\end{tabular}

volatility, the power of WT, PV, and LD is subject to uniform distribution with a $\pm 10 \%$ deviation of the calculated or forecasted values.

Table 3 lists the parameters of CG. The rated capacity of BT is $90 \mathrm{kWh}$, while its initial capacity is saturated. The parameters of $k=2.2, c=15, \alpha=0.45$, and $\beta=9.18$ are calculated according to Sections 3.1 and 3.2. Figs. 1(a) and 1(b) illustrate the forecasting power of WT, PV, and LD.

The parameters of PSO are set as follows: number of particles $=250$; maximum number of iterations $=300$; maximum fly speed $=1$; minimum flying speed $=-1$; maximum position $=5$, minimum position $=-5$, inertia weight $=\mathrm{C} 1=1.3$ and $\mathrm{C} 2=2.8$; and number of simulations $=$ 6000 .

\subsection{Results and analysis}

\subsubsection{Optimization results of the two operation modes}

In the first operation mode, the randomness power of PV, WT, and LD is not considered. The objective function is then transformed into the following deterministic formula:

$$
\begin{gathered}
\min \bar{F}=\min \left[\sum_{t=1}^{24} \sum_{j=1}^{3} C_{O M j}\left(P_{t j}\right)+C_{f t j}\left(P_{t j}\right)\right. \\
\left.+C_{\text {entj }}\left(P_{t j}\right)+C_{\mathrm{var} t j}\left(P_{t j}\right)\right]
\end{gathered}
$$

The spinning reserve constraint is also transformed into the following deterministic expression:

$$
\left(P_{D G t}+P_{M T t}+P_{F C t}+P_{P V t}+P_{W T t}+P_{B T \max t}\right)-P_{L D t} \geq P_{S R t}
$$

The economic dispatch of the first mode will be optimized by POS.

The second operation mode is the uncertainty model proposed in this paper. The confidence levels are taken as $\beta_{1}=0.96$ and $\beta_{2}=0.95$.

Fig. 2 shows the cost result of $C G$ in the two modes. Such cost changes per iteration. The economic dispatch costs of CG in the deterministic and uncertainty models are $\$ 636.1862$ and $\$ 597.8578$, respectively. The deterministic model is just the same as the uncertain mode with $\beta_{1}=\beta_{2}=1$, that is, the objective function and constraint condition are tightened, thereby increasing the operation costs.
Table 4. Optimization results under different confidence levels

\begin{tabular}{c|c|c|c|c}
\hline \multirow{2}{*}{$\beta_{2}$} & \multicolumn{4}{|c}{ The minimum operation costs of CG $(\$)$} \\
\cline { 2 - 5 } & \multicolumn{4}{|c}{$\beta_{1}$} \\
\cline { 2 - 5 } & 0.98 & 0.94 & 0.90 & 0.85 \\
\hline 0.98 & 630.2867 & 623.0548 & 607.5481 & 589.2846 \\
\hline 0.94 & 622.1248 & 616.2547 & 600.2153 & 581.5795 \\
\hline 0.90 & 599.0481 & 595.6549 & 582.0134 & 570.1548 \\
\hline 0.85 & 584.9540 & 571.1248 & 569.2001 & 545.2647 \\
\hline
\end{tabular}

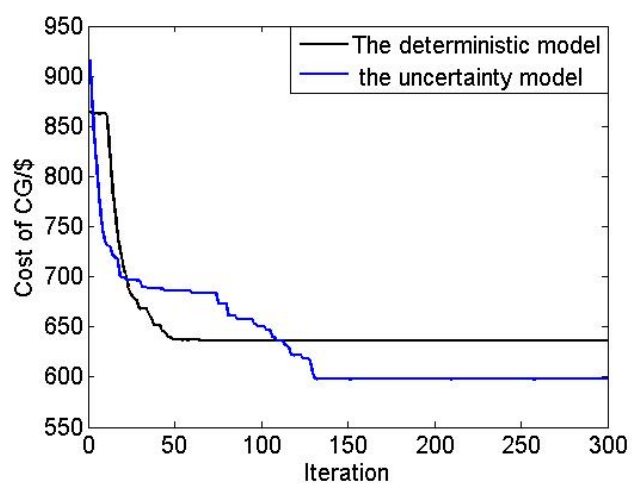

Fig. 2. Variation of CG cost and iteration in two modes

\subsubsection{Optimization results of different confidence levels}

Table 4 lists the optimization results at different confidence levels. The minimum operation cost of CG will change with different $\beta_{1}$ and $\beta_{2}$, and will increase along with $\beta_{2}$. The physical significance of $\left(1-\beta_{2}\right)$ indicates the stability of the system and is reflected in the maximum probability of allowing loss of load. In other words, a higher stability of the system corresponds to higher operation costs of CG. The appropriate confidence levels of $\beta_{2}$ must be selected in the actual operation to balance the stability and economy of MG. At the same time, the operation costs of $\mathrm{CG}$ will increase along with the confidence levels of $\beta_{1}$ and under the same confidence levels of $\beta_{2}$. The actual operating costs include power adjustment costs, and the growth of such cost will accelerate along with the increase of $\beta_{1}$. This finding reflects the meaning of $\beta_{1}$, that is, the minimum probability of a successful power adjustment.

\subsubsection{Effect of optimization results by BT}

The residual capacity of BT is checked before each dispatch. If the residual capacity of BT is less than $20 \%$ of its rated capacity, then the BT will charge instead of output. Otherwise, the BT will output in economic dispatch until its capacity reaches $20 \%$ of its rated capacity.

Fig. 3 presents the economic dispatch of MG with and without BT under the same parameters. The operation costs of CG with and without BT are \$654.3352 and \$703.2557, respectively. Obviously, the output of BT reduces the 


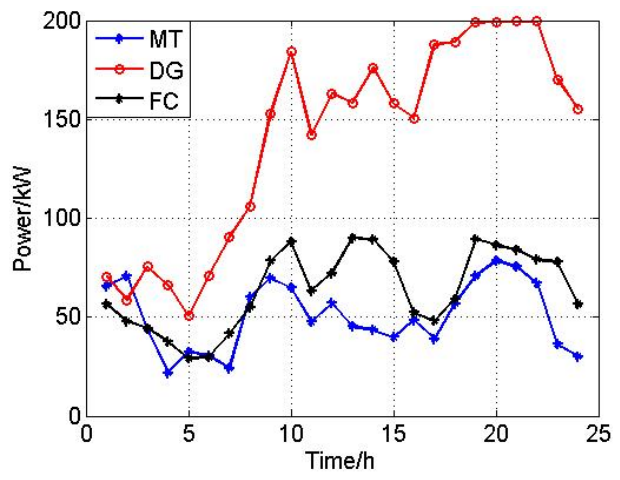

(a) the MG without BT

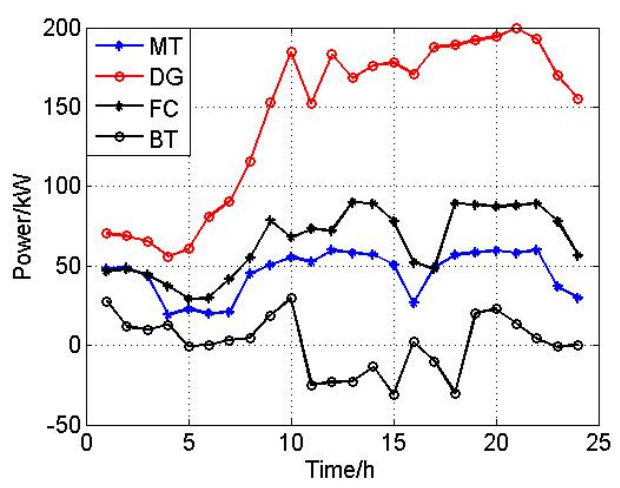

(b) the MG with BT

Fig. 3. Costs of CG with and without BT

output of the other CG, thereby reducing the costs of CG.

\subsubsection{Limitations of the proposed method}

To achieve a statistically meaningful distribution, the MC simulation must run a large number of iterations, and the PSO must be performed for each realization of the parameters in the simulation. This process requires a long computation time and can affect the actual operations.

\section{Conclusion}

To consider the influence of the randomness of the power of PV, WT, and LD, this paper proposed a dynamic economic dispatch model of MG based on CCP. The probability constraint concerning $\mathrm{MG}$ reliability was appended in the model. A confidence level was set to the spinning reserve constraint to meet the system requirements, and the minimum cost of $\mathrm{CG}$ was taken as the objective function that should be no less than a specified confidence level. MC simulation and PSO were applied to optimize the model. An island MG that includes PV, WT, DG, MT, FC, and BT and operates on a single day was taken as an example. The effect of confidence levels on the system operation costs and two operation modes of the MG were also discussed. The optimization method has several limitations, such as long time consumption and multiple iterations. Future studies can propose and test additional methods to address these limitations.

\section{Acknowledgements}

This work was supported by National Natural Science Foundation of China (Grant number: 61561007).

\section{References}

[1] Islam, Md. R. and Gabbar, H. A. "Study of Small Modular Reactors in Modern Microgrids," Int T Electr Energy, vol. 25, no. 9:1943-1951, 2015.

[2] Venkataramanan Giri. Marnay Chris. "A Larger Role for Microgrids," IEEE Power Energy M, vol. 6, no. 3:78-82, 2008.

[3] Xiarnay Chris,Asano Hiroshi, Papathanassiou Stavros, et a1. "Policy Making for Microgrids," IEEE Trans. Energy Convers, vol. 6, no. 3:66-77, 2008.

[4] Faisal A. Mohamed, Heikki N. Koivo. "System modelling and online optimal management of MicroGrid using mesh adaptive direct search," Int $J$ Elec Power, vol. 32, no. 5: 398-407, 2010.

[5] Si Young Lee, Young Gyu Jin, Sun Kyo Kim,et al. "Operation Planning of Reserve in Microgrid Considering Market Participation and Energy Storage System," J Electr Eng Technol, vol. 9, no. 3: 10891095, 2014.

[6] Ango Sobu, Guohong Wu Member. “Optimal Operation Planning Method for Isolated Microgrid Considering Uncertainties of Renewable Power Generations and Load Demand," IEEE PES ISGT Asia, 1569536541:1-6, 2012.

[7] Sung-Eun Lee, Dong-Jun Won and Il-Yop Chung, "Operation Scheme for a Wind Farm to Mitigate Output Power Variation," J Electr Eng Technol, vol. 7, no. 6: 869-875, 2012.

[8] Hak-Man Kim, Tetsuo Kinoshita and Yujin Lim, "Talmudic Approach to Load Shedding of Islanded Microgrid Operation Based on Multiagent System," J Electr Eng Technol, vol. 6, no. 2, pp: 284 292, 2011.

[9] Ma Xiyuan, Wu Yaowen, Fang Hualiang, et al . "Optimal Sizing of Hybrid Solar-wind Distributed Generation in an Islanded Microgrid Using Improved Bacterial Foraging Algorithm," Proceedings of the CSEE, vol. 31, no. 25:17-26, 2011.

[10] Yiwei Ma, Ping Yang, Hongxia Guo,et al. "Dynamic Economic Dispatch and Control of a Stand-alone Microgrid in DongAo Island," J Electr Eng Technol, vol. 10, no. 4: 1432-1440, 2015.

[11] Daizheng Huang, Renxi Gong, Shu Gong. "Constrained Multiobjective Optimization for Microgrid Based on Nondominated Immune Algorithm," IEEJ 
Trans, vol. 10, no. 4: 376-382, 2015.

[12] Xufeng $\mathrm{Xu}$, Joydeep Mitra, Tingting Wang,et al. "Reliability Evaluation of a Microgrid Considering Its Operating Condition," J Electr Eng Technol, vol. 11, no. 1: 47-54, 2016.

[13] Wang Rui, Gu Wei, Wu Zhi. "Economic and Optimal Operation of a Combined Heat and Power Microgrid," Automation of Electric Power Systems, vol. 35, no. 8:22-27, 2011.

[14] Gu Wei, Wu Zhi, WANG Rui. "Multi-objective Optimization of Combined Heat and Power Microgrid Considering Pollutant Emission," Automation of Electric Power Systems, vol. 36, no. 14:177-185, 2012.

[15] Chen Jie, Yang Xiu, Zhu Lan, et al. "Microgrid multiobjective economic dispatch optimization," Proceedings of the CSEE, vol. 33, no. 19:57-67, 2013.

[16] Liu Xiaoping, Ding Ming, Zhang Yingyuan, et al. "Dynamic economic dispatch for microgrids," Proceedings of the CSEE, vol. 31, no. 31:77-84, 2011.

[17] Baoding Liu. "Uncertainty Theory (Fourth Edition)," Department of Mathematical Sciences, Tsinghua University, Beijing, China. Available at: http://orsc.edu.cn/liu

[18] Nikos Hatziargyriou, Hiroshi Asano, Reza Iravani, et al. "Microgrids," IEEE Power Energy M: 78-94, 2007.

[19] Lasseter R H, Akhil A, Marnay C, et a1. "Integration of Distributed Energy Resources: the CERTS Microgrid Concept," USA-Consortium for Electric Reliability Technology Solutions, 2002.

[20] Zhi Wu, Wei Gu, Rui Wang, et al. "Economic Optimal Schedule of CHP Microgrid System Using Chance Constrained Programming and Particle Swarm Optimization," IEEE-PES General Meeting, July 2011.

[21] Mohamed, F., and Heikki, koivo. "System Modelling and Online Optimal Management of Microgrid," 6th International Workshop on Large-Scale Integration of Wind Power and Transmission Networks for Offshore Wind Farms: 26-28, 2006.

[22] Mohamed F A, Koivo H. N. "Online Management of Microgrid with Battery Storage Using Multiobjective Optimization," Proceedings of 2007 International Conference on Power Engineering, Energy and Electrical Drives. Setubal, Portugal : IEEE:231-236, 2007.

[23] Qian Kejun, Yuan Yue, Shi Xiaodan, et al. "Environmental Benefits Analysis of Distributed Generation," Proceedings of the CSEE, vol. 28, no. 29:11-15, 2008.

[24] Yang Hongxing, Zhou Wei, Lu Lin, et al. "Optimal Sizing Method for Stand-alone Hybrid Solar-wind System with LPSP Technology by Using Genetic Algorithm," Solar Energy, vol. 82, no. 4:354-367, 2008.

[25] Bowden G J, Barker P R, Shestopal V O, et al. "Weibull Distribution Function and Wind Power
Statistics," Wind Engineering, vol. 7, no. 2:85-98, 1983.

[26] He Xianfu, Lu Xia, Yang Yuejin Liu Wankun. "Design, manufacture and operation for wind turbine," Chemical Industry Press, Beijing, China, 2009.

[27] NREL. "Energy modeling software for hybrid renewable energy systems," Available at: http://www.homerenergy.com/,2013.

[28] Zhang Meixia, Chen Jie, Yang Xiu, et al. "Threephase Power Flow Calculation for Microgrid with Volatile Wind Power, Photovoltaic Power and Load," Proceedings of the CSEE, vol. 33, no. 13:101-107, 2013.

[29] Karaki S H, Chedid R B, Ramadan R. "Probabilistic Performance Assessment of Autonomous Aolar-wind Energy Conversion Systems," IEEE T Energy Conver, vol. 14, no. 3:766-772, 1999.

[30] Liang Shuang, Hu Xuehao, Zhang Dongxia, et al. "Probabilistic Models Based Evaluation Method for Capacity Credit of Photovoltaic Generation," Automation of Electric Power Systems, vol. 36, no. 13:32$36,2012$.

[31] Huang Haiyun. "Principles of application of solar photovoltaic power generation," Chemical Industry Press, Beijing,China,2009.

[32] Lin Shaobo, Han Minxiao, Zhao Guopeng, et al. "Capacity Allocation of Energy Storage in Distributed Photovoltaic Power System Based on Stochastic Prediction Error," Proceedings of the CSEE, vol. 33, no. 4:25-34, 2013.

[33] Yang Yun, Zhang Shijie, Xiao Yunhan, et, al. "Application of Monte Carlo Method in Uncertainty Evaluation for Cogeneration Systems," Proceedings of the CSEE, vol. 33, no. 2:16-23, 2013.

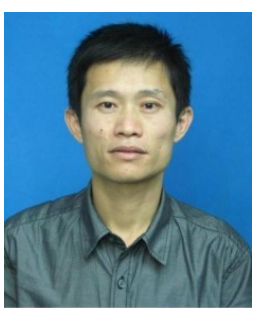

Daizheng Huang He received a M.S. degree in optical engineering from Huazhong University of science and technology in 2006, and received a Ph.D degree in electric power system and automation from Guangxi University in 2015. He now research interests are artificial intelligence algorithm.

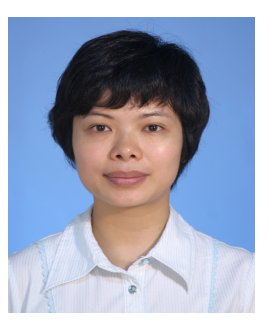

Ling-ling Xie She was born in Guangxi Province, China, in 1980. And received her B.S, M.S and Ph.D. in automation, control science and engin-eering and electric power system and automation from Guangxi University, China, in 2003, 2006 and 2012. She is currently an associate professor at Electrical Engineering School of Guangxi University. Her main 
research interests include nonlinear dynamics, chaos control and bifurcation analysis in power electronics.

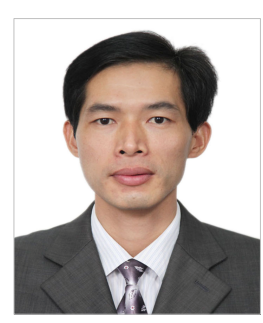

Zhihui Wu was born in 1974 and received his B.S degree in physics from Guangxi Normal University, China in 1998, and received a M.S degree in physiology from Guangxi Medical University in 2010, China. He is currently associate professor in Guangxi Medical University. He now research interest is electronics. 